livraisons

d'Histoire

de l'Architecture

\section{Livraisons de l'histoire de l'architecture}

$18 \mid 2009$

D'architectures

\title{
Un néo-gothique fantastique : Antoine-Marie Chenavard et la cathédrale de Belley
}

A fancy neo-gothic: Antoine-Marie Chenavard and the Belley cathedral Fantastisches im Neogotikstil : Antoine-Marie Chenavard und die Kathedrale von Belley

\section{Philippe Dufieux}

\section{OpenEdition}

Journals

Édition électronique

URL : http://journals.openedition.org/lha/226

DOI : $10.4000 /$ /ha.226

ISSN : 1960-5994

\section{Éditeur}

Association Livraisons d'histoire de l'architecture - LHA

Édition imprimée

Date de publication : 10 décembre 2009

Pagination : 77-92

ISSN : 1627-4970

\section{Référence électronique}

Philippe Dufieux, « Un néo-gothique fantastique : Antoine-Marie Chenavard et la cathédrale de Belley

", Livraisons de l'histoire de l'architecture [En ligne], 18 | 2009, mis en ligne le 10 décembre 2011,

consulté le 01 mai 2019. URL : http://journals.openedition.org//ha/226 ; DOI : 10.4000/lha.226

Ce document a été généré automatiquement le 1 mai 2019.

Tous droits réservés à l'Association LHA 


\title{
Un néo-gothique fantastique : Antoine-Marie Chenavard et la cathédrale de Belley
}

\author{
A fancy neo-gothic: Antoine-Marie Chenavard and the Belley cathedral \\ Fantastisches im Neogotikstil : Antoine-Marie Chenavard und die Kathedrale \\ von Belley
}

Philippe Dufieux

«Je sens toute l'importance qu'il y a de conserver
aux monuments des arts leur caractère primitif et
même leur couleur que le temps seul peut donner,
parce que c'est elle qui produit sur nous la
première impression et qu'elle ajoute à l'édifice un
charme de plus en accusant son antiquité " ${ }^{1}$.

1 L'allégeance passionnée d'Antoine-Marie Chenavard (1787-1883) à la culture classique a longtemps occulté ses travaux en matière de restauration monumentale, lui qui compte pour l'une des figures majeures du gothique troubadour en France dont la cathédrale de Belley (1836-1855), premier essai de cathédrale idéale avant les entreprises archéologiques de Lassus et de Viollet-le-Duc, constitue l'une des réalisations les plus emblématiques. En ce domaine pourtant, son talent connut des fortunes diverses au point que ses biographes n'eurent de cesse de nuancer les critiques acerbes dont ses réalisations firent l'objet de son vivant même. En avocats de leur maître, Antonin Louvier et Adolphe Coquet plaident l'indulgence : "Ce sentiment religieux que nous cherchons dans nos temples, l'art antique dans ses règles immuables, ne pouvait pas le lui donner [... ] et l'on pourra excuser Chenavard, si dans les conditions où il se trouvait, il n'a pas compris ce style gothique qu'on qualifiait alors de barbare ${ }^{2}$ ». Clair Tisseur évoque à peine ses travaux d'art médiéval au risque d'entacher sa gloire posthume ${ }^{3}$, tandis que pour Roidot, ses œuvres néo-gothiques et classiques participent d'une même pensée, «la science des formes ogivales se trouve comme épurée par les souvenirs de l'art grec », en d'autres termes, "une idée grecque sous une physionomie gothique ${ }^{4}$; en écho à la 
problématique chère à Viollet-le-Duc selon laquelle la rationalité antique se serait transmise aux bâtisseurs du Moyen-Âge. Peu après sa mort, L'Écho de Fourvière ne manquera pas de déplorer combien ses restaurations témoignent « de son peu d'étude dans l'art ogival »5. Quarante ans plus tôt, en pleine querelle entre les » gothiques » et les " classiques », Chenavard sera cité dans les Annales archéologiques aux côtés de Soufflot et de Quatremère de Quincy comme l'un des pires vandales que les églises de France aient connues ${ }^{6}$. Bien qu'il n'ait jamais été élevé à l'art médiéval, Chenavard n'en demeure pas moins attentif au début de sa carrière au charme pittoresque des monuments en faisant valoir une approche plus volumétrique que structurelle de l'architecture gothique. Alors que les maîtres d'œuvre formés dans la lignée de Boullée et de Ledoux ne rêvent que de monuments euclidiens d'un classicisme rigoureux, dans les premières décennies du XIX ${ }^{\mathrm{e}}$ siècle, nombre d'architectes durent se former eux-mêmes à l'art médiéval en l'absence des contributions littéraires et archéologiques qu'allaient bientôt offrir les années 1830-1840. Si Chenavard réalise quelques travaux néo-classiques à son retour de Rome en 1818, le Grand théâtre de Lyon mis à part, l'essentiel de son activité des années 1820-1830 touche précisément à des chantiers de restauration monumentale auxquels il convient d'ajouter la reconversion des ensembles conventuels. En 1831, à l'occasion de son discours de réception à l'Académie royale de Lyon, l'architecte condamnera sans appel l'engouement contemporain pour l'archéologie médiévale: «Peut-on ne pas s'effrayer pour le bon goût de cet amour du gothique dont les formes hétérogènes se montrent jusque dans nos ameublements [...] ? Quel rapport y a-t-il entre nos mœurs actuels, notre littérature, nos progrès dans les arts du dessin, et le style barbare d'un vitrail gothique flanqué de deux statues, ébauches informes, sans proportion, accompagnés d'inscriptions dont les caractères tudesques déchirent l'œil et dont le langage est à peine compris ?7. » "Rien ne parait aussi nouveau que l'antique ", écrivait-il en conclusion de ce vibrant plaidoyer en faveur de l'étude de l'art classique, en digne émule de Quatremère de Quincy et de Winckelmann ${ }^{8}$. À cette date, une telle position de principe ne supporte apparemment aucune nuance même si l'architecte prend soin de passer sous silence ses premières expériences néo-médiévales. Cette déclaration intervient, il est vrai, près d'une dizaine d'années avant les grands débats qui devaient opposer les gothiques et les classiques sur la question de savoir quel est l'art le plus authentiquement chrétien. À partir de 1836, l'architecte conduit successivement la restauration de l'église SaintVincent de Chalon-sur-Saône (Saône-et-Loire, 1826-1827), l'agrandissement de la cathédrale de Belley (Ain, 1836-1851) et de celle de Viviers (Ardèche, 1827-1839), l'achèvement de l'église Saint-Étienne de Roanne (Loire, 1837-1843). À ces chantiers, s'ajoutent ceux qui relèvent du domaine diocésain de Lyon, sans oublier de nombreux travaux paroissiaux dans le Rhône.

\section{Belley, premier essai de cathédrale idéale}

2 Rendue au culte par le Concordat de 1802, mais comme simple église paroissiale, l'ancienne cathédrale de Belley est encore rattachée à cette date, comme le département de l'Ain, au diocèse de Lyon qui, taillé au début du XIX siècle dans les anciennes circonscriptions ecclésiastiques à l'échelle des ambitions de son éminent archevêque, le cardinal Joseph Fesch, réunit alors le Rhône, la Loire et l'Ain. Cet ensemble forme alors le diocèse le plus peuplé de France avec près 1200000 âmes. À l'issue d'âpres négociations, le diocèse de Belley est restauré en octobre 1822 et son premier évêque, Mgr Alexandre 
Romain Devie est promptement nommé trois mois plus tard, le 13 janvier 1823. Fervent zélateur du néo-gothique, le nouvel évêque de Belley s'intéresse de près aux questions archéologiques et de convenance liturgique comme en témoigne la publication par ses soins du Rituel de Belley qui compte pour l'un des premiers manuels de recommandations à destination des ecclésiastiques 9 . La reconstruction de la vieille église Saint-Jean-Baptiste de Belley se confondra avec celle du diocèse, œuvre immense dont Mgr Devie sera le maître d'œuvre. Sous le gouvernement de cet évêque bâtisseur (1823-1852), pas moins de trois cents églises sont restaurées tandis que cinquante églises nouvelles sont construites ${ }^{10}$. L'achèvement de la cathédrale de Belley doit marquer la renaissance du diocèse et Mgr Devie s'inquiète très tôt de l'état de délabrement de l'édifice, une église sans unité de style, composée d'un chœur gothique élevé vers 1480 accolé à une nef romane découronnée de ses deux clochers à la Révolution: "L'église cathédrale de Belley présente plusieurs genres d'architecture - écrivait l'évêque de Belley au ministre des cultes en avril 1829 - mais le chœur, construit dans un genre gothique avec élégance, forme la seule partie que l'on puisse espérer conserver [...] $»^{11}$. Dans quel style faut-il alors reconstruire la façade et les clochers ? C'est un problème épineux auquel l'architecte Jean Pollet (11 floréal an III-1839) se heurtera dans ses premières propositions, dont l'une dite «byzantine » [romane], sans jamais remporter l'assentiment de l'administration des cultes ni celui du conseil des bâtiments civils qui émet de sérieuses réserves: " $\mathrm{La}$ construction dont il s'agit est assez importante pour devoir être développée et expliquée plus complètement", écrit l'inspecteur général Tardieu en juin $1829^{12}$. Devant les hésitations de Pollet, en avril 1830, Chenavard est sollicité en vue de collaborer à la définition du projet, «architecte [...] ayant déjà fait preuve de talent et de capacité dans des restaurations de même genre ", écrivait le ministre des affaires ecclésiastiques et de l'instruction publique au préfet de l'Ain dans une allusion à peine voilée à l'achèvement de la cathédrale de Chalon-sur-Saône quelques années plus tôt ${ }^{13}$. Mais leur profonde mésentente embarrasse Mgr Devie: «Des différents intervenus entre les deux hommes rendent impossible toute communauté de travail entr'eux [sic] ", écrivait-il en octobre $1832^{14}$. En février de l'année suivante, Pollet est dessaisi du chantier au profit de son confrère. En octobre 1833, Chenavard établit un nouveau projet inspiré de la façade étudiée pour l'église Saint-Étienne de Roanne et attire l'attention de l'administration sur la nécessité d'insérer les maçonneries de l'édifice par des tirants métalliques en raison des profonds désordres causés par le tremblement de terre qui eut lieu le 22 février 1822 à Belley et dont les secousses ont dangereusement fragilisé l'édifice. À l'origine, il n'est question que de reconstruire la façade en démolissant notamment le clocher en bois construit au début du XIXe siècle dont l'architecte ne peut que constater l'état de ruine, mais la restauration partielle de la cathédrale amène bientôt une reconstruction totale. Chenavard étudie à cette fin un projet d'ensemble comprenant la reconstruction des nefs : «faisant suite à l'architecture gothique du chœur " $^{15}$. Dans l'attente, la façade romane et le clocher provisoire sont démolis à la fin de l'année 1835 et les travaux de fondation de la nouvelle façade s'exécutent jusqu'en mai de l'année suivante, date à laquelle Chenavard se rend sur place et s'inquiète de la stabilité des nefs, "Cette démolition ayant mise à découvert la maçonnerie intérieure, je vis qu'au défaut de base solide s'ajoutait une construction des plus vicieuses [...]. Les nefs latérales qui ordinairement offrent une résistance aux efforts latéraux des voûtes des grandes nefs ne leur prêtent ici aucun appui car, dit-il, les murs des arcs-doubleaux des bas-côtés s'arrêtent aux voûtes et ne s'élèvent pas suivant la pente du comble jusqu'au droit de la retombée de la grande voûte ${ }^{16}$. » L'architecte cite à ce sujet, dans un courrier adressé au 
préfet de l'Ain en juin 1836, de larges extraits de précédents rapports détaillant l'état de dégradation de l'édifice et ses problèmes de stabilité mais, assez curieusement, il n'est jamais fait mention de l'origine de ce désordre alors que la charpente du comble est précisément reconstruite en 1826. Chenavard avance à ce sujet un argument décisif, " avant la démolition, on ne pouvait reconnaître [ce problème], car il n'existe dans le comble aucune ouverture pour pénétrer au-dessus des bas-côtés ». S'agit-il véritablement d'un défaut de conception de la part des constructeurs romans, ou Chenavard se révèle-til incapable de résoudre ce problème de stabilité ? Toujours est-il que l'architecte propose la démolition complète des nefs. À sa décharge, il est vrai que ce type d'intervention entraine, en matière de raccordement, des problèmes techniques souvent mal résolus qui aboutissent fréquemment à la reconstruction pure et simple, «Je pense, Monsieur le Ministre que la reconstruction de la nef et des bas-côtés est devenue indispensable, les causes en sont évidentes ", conclut-il d'un ton assuré en rappelant que le projet général envisageait déjà cette solution. L'affaire traîne en longueur, Chenavard se montre de plus en plus insistant, mais la commission des arts et des édifices religieux lui fait observer que ses dessins sont peu satisfaisants et qu'ils ne donnent qu'une faible idée de l'état actuel du monument. En matière de restauration monumentale, la prudence devrait toujours prévaloir selon Caristie, rapporteur au conseil des bâtiments civils: «Cette démolition ne saurait être justifiée par le seul désir de régulariser l'ensemble du monument ", écrivait celui-ci en décembre $1839^{17}$, avant d'ajouter que la mission des architectes est de conserver et non de créer ou d'améliorer: "L'intérêt archéologique exige que l'on reproduise fidèlement ce qui existait, agir autrement, c'est dénaturer un monument, c'est en changer le caractère ». Cette position de principe est cependant immédiatement nuancée. De l'avis même de Caristie, «lorsque les constructions anciennes ont fait leur temps, que leur reconstruction nuirait à l'ensemble et à l'unité que doit présenter tout édifice, il faut se résoudre à en faire le sacrifice ". Dès lors, l'unité de style devait-elle l'emporter sur la conservation et le strict respect de l'archéologie avec l'aval du conseil des bâtiments civils qui observe d'ailleurs combien le projet de reconstruction de Chenavard est judicieusement conçu. Afin de couper court aux critiques, l'architecte fait valoir la conservation d'un portail latéral roman et insiste sur le fait qu'il s'emploiera simplement à continuer l'édifice sur le modèle du chœur. Le directeur de l'administration des cultes, soucieux de l'emploi des fonds publics, ne partage pas ce point de vue et s'interroge quant aux véritables intentions de l'architecte en demandant expressément la conservation des nefs au nom de l'art et de l'économie : "Un pareil projet serait contre toutes les règles reçues aujourd'hui » ${ }^{18}$. Le Courrier de l'Ain s'empare bientôt de l'affaire (20 septembre 1838) et accuse les pouvoirs publics d'engager un projet qui entraînerait la destruction des $4 / 5^{\mathrm{e}}$ de l'édifice. Le préfet de l'Ain et le ministre des cultes somment l'architecte de répondre à ses détracteurs et ce dernier s'exécute promptement. Accusé d'avoir laissé se dégrader l'édifice à l'issue de la destruction de la façade, Chenavard cite à ce sujet l'extrait d'un rapport adressé au ministre par l'un de ses contempteurs : «On s'est plu à en exagérer la dégradation afin d'être autorisé à le détruire ", lit-on sous la plume de Ferdinand de Guilhermy ${ }^{19}$. Une accusation calomnieuse selon le maitre d'œuvre de la cathédrale, «La démolition de cet édifice était donc nécessaire ; j'ai démontré, écrit Chenavard, que, sous le rapport de l'art et de l'histoire, il était sans intérêt, et que dans son état de ruine les travaux de consolidation n'auraient eu de résultat que de le rendre plus difforme à la vue et d'absorber en pure perte des sommes considérables ${ }^{20}$. " Le thème de l'incompétence de l'opinion locale est largement invoqué pour justifier la nature d'une transformation 
radicale autorisée par l'administration, ce que ne manque pas de rappeler à plusieurs reprises Chenavard. Le souvenir des diatribes dont il avait fait l'objet quelques années plus tôt à Lyon reste visiblement présent à son esprit. L'affaire s'envenime et Chenavard peine à se justifier, « Le même esprit de critique aveugle et malveillant s'étend de la part de M. de Montalembert sur les restaurations de la cathédrale de Lyon; son opinion ne peut balancer la mienne et je m'en réfère à des juges plus compétents que lui qui les approuvent ", écrit-i ${ }^{21}$. Si l'architecte réalise ses desseins à Belley, la bataille de l'opinion est perdue. Chenavard ne s'embarrasse pas de scrupules, la question de la conservation des nefs romanes ne semble pas même se poser. À peine s'emploie-t-il à reproduire, par symétrie, au transept sud de la cathédrale, le motif formé par une galerie aveugle d'époque romane, conservée au transept nord selon l'idée que l'édifice doit impérativement recouvrir son unité stylistique, gothique en l'occurrence : "Quel que soit le style d'architecture d'un édifice, nous pensons qu'il y a nécessité de s'y conformer soit dans l'exécution de son complément, soit dans la restauration des parties détruites, ici l'artiste qui succède doit faire abnégation de ses propres goûts et consentir à suivre religieusement la marche tracée par le premier auteur ", écrivait-il en $1842^{22}$. Dans cet esprit, l'architecte généralisera à toutes les parties de la construction le dessin des travées du chœur (ill. 4), qu'il s'agisse de l'avant-chœur comme des nouvelles nefs qui se poursuivent sur cinq travées après le transept; un imposant clocher porche en façade achève la composition (ill. 1).

\section{1 : Cathédrale de Belley (Ain), façade principale}

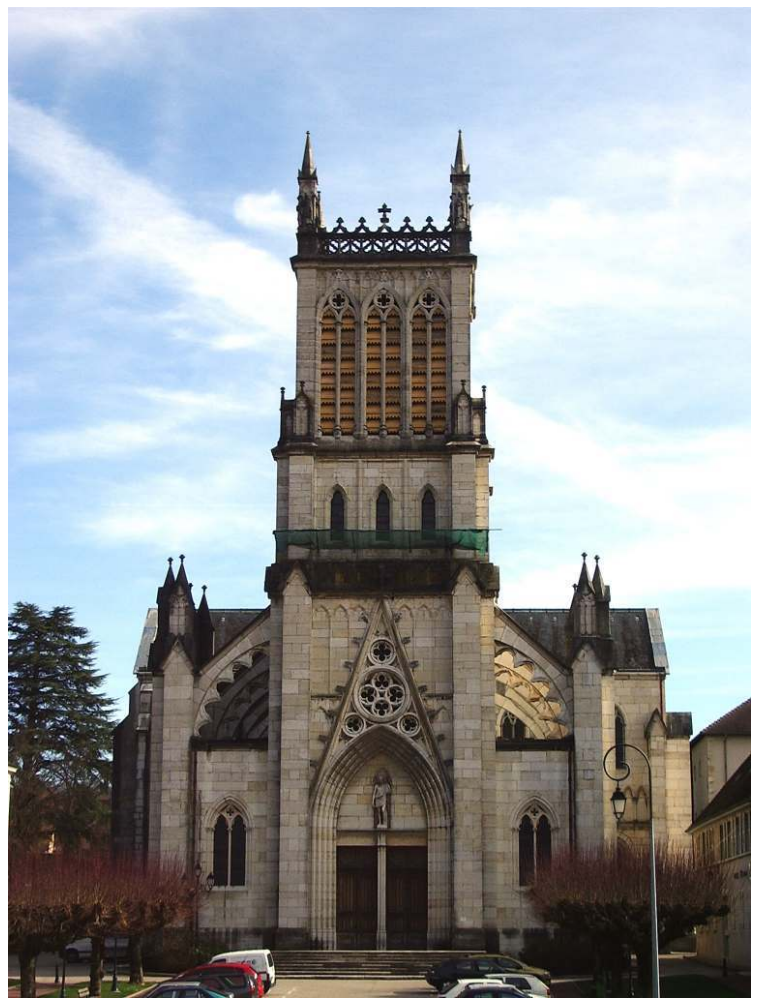

Cl. Jean-Christophe Stuccilli 
III. 4 : Cathédrale de Belley (Ain), détail des travées construites par Chenavard

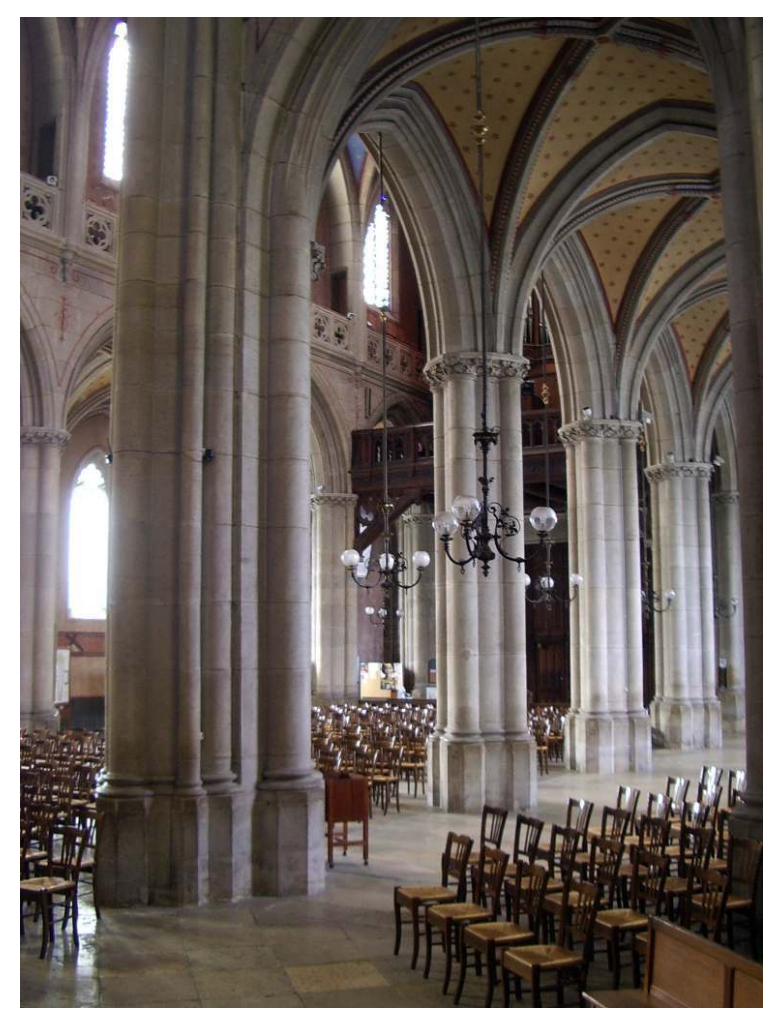

Cl. Philippe Dufieux

3 Le faible développement des bas-côtés ne permettant pas de composer une façade harmonique, à l'image de celle qui avait été élevée à Chalon-sur-Saône quelques années plus tôt, Chenavard cherchera à corriger l'impression massive du clocher en soulignant les verticales qui peinent à hérisser la silhouette de la tour ; le bandeau horizontal qui termine le couvrement des bas-côtés ruinant à lui seul cette tentative. Ajoutons à cela que l'absence de flèche accuse plus encore la physionomie anglaise de l'édifice, proche par l'esprit du gothique perpendiculaire, ce gothique insulaire qui devait profondément influencer la culture romantique et fantastique contemporaine. Si la reproduction d'un module à l'échelle d'un édifice entier ne remet pas en cause la lecture de la construction médiévale par le maître d'œuvre, la reconstruction des arcs-boutants de la chapelle axiale suscite son incompréhension (ill. 3). 


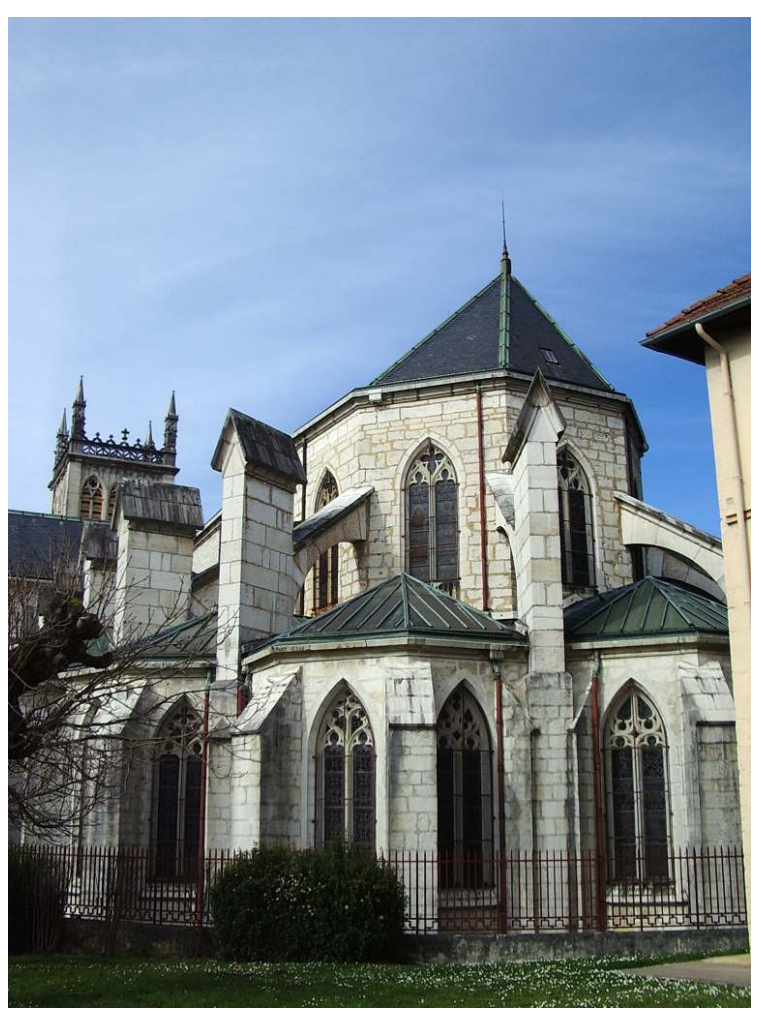

Cl. Jean-Christophe Stuccilli

Le système de contrebutement de l'abside flamboyante a connu de nombreuses transformations par le passé et l'architecte en est réduit à des hypothèses : «Lequel de ces contreforts faudra-t-il prendre pour modèle, car ils diffèrent entre eux de hauteur et de forme ", s'interroge-t-il en avril $1850^{23}$. Ses diverses propositions seront rejetées successivement, «Les arcs-boutants ne contrebutent nullement les grandes voûtes. Ils viennent porter beaucoup au-dessus de la poussée des voûtes. Ils sont appareillés d'une manière vicieuse. Le projet général n'a pas été étudié avec une attention suffisante ", liton dans un rapport daté du 2 septembre $1848^{24}$. Le jugement de Viollet-le-Duc est sans appel: "L'architecte chargé de ces travaux n'a pas une connaissance intime des monuments qu'il cherche à imiter et que, bien qu'il essaye d'en suivre la forme avec plus ou moins de goût, il n'en comprend pas la construction sans laquelle la forme n'a aucune signification ${ }^{25}$. Le 20 juin 1850, la commission des arts et des édifices religieux reprend les conclusions de Viollet-le-Duc à la vue des restaurations conduites au chevet: "Les contreforts de l'abside non seulement sont lourds et d'un mauvais effet mais ce qui est beaucoup plus grave, sont en opposition avec les indications qui existent encore de leur état primitif. C'est en vain qu'on ferait observer que les contreforts de la nef ont été restaurés conformément au dessin présenté et qu'on se fonderait sur ce précédent pour déterminer dans un but d'unité et d'harmonie la restauration de ceux de l'abside sur le même plan ». Avant de conclure : "La question d'art et de goût mise à part, aucune considération ne doit faire sacrifier la vérité historique dans la restauration d'un ancien monument $»^{26}$. Alors que les critiques se multiplient, Chenavard fait valoir contre toute attente qu'il s'est montré soucieux de préserver le caractère archéologique du monument: «Il [l'architecte] a fait preuve de son esprit de conservation, en luttant 
contre les avis, pour conserver des parties fort délabrées et en désaccord avec le style du sanctuaire, mais précieuses à ses yeux parce qu'elles portaient le cachet d'une époque antérieure et pouvait servir à l'histoire du monument ${ }^{27}$. » De tels scrupules, s'exprimant près de quinze ans après le début du chantier, ne manquent pas de surprendre. Chenavard est acculé à la démission: «Permettez-moi M. le Ministre d'insister sur ce point, écrit-il, parce que c'est une opinion défavorable que rien ne justifie, que je n'ai cessé de combattre et que pourtant la commission des édifices religieux reproduit incessamment et sans preuve [...]. J'ai conservé et non mutilé $»^{28}$. Mais en 1850 , les travaux sont visiblement trop avancés pour envisager une restauration plus archéologique de l'édifice, ce que reconnaît incidemment le ministre des cultes, « Après les nombreuses et regrettables déformations qu'a subies le monument, il y a paru qu'il serait peut-être inutile de chercher aujourd'hui à revenir à un système de restauration uniforme et régulier $»^{29}$. En 1842, la nouvelle façade et le clocher sont terminés tandis que les nefs et l'avant-chœur s'élèvent entre 1840 et 1851 . Le 12 janvier 1851, la nouvelle cathédrale est ouverte au culte. En un quart de siècle, l'État aura dépensé plus de 800000 frs pour sa reconstruction ce qui en fait l'un des chantiers diocésains les plus coûteux engagé sous la Monarchie de Juillet. «J'ignore d'où provient cette opposition à mon égard et la critique d'une connaissance peu approfondie des monuments religieux du Moyen Âge. C'est ce que ne démontrent ni l'église paroissiale de Saint-Étienne à Roanne que j'ai construite de 1837 à 1843, ni même la cathédrale de Belley. Qu'il me soit permis d'ajouter que trente années d'études de l'architecture et un long professorat à l'École des beauxarts de Lyon m'ont rendu familier dans la comparaison des styles, l'observation des caractères aux différentes époques et l'appréciation des rapports des parties entre elles ", écrit Chenavard en juillet 1848, conscient que sa notoriété est sérieusement mise à mal par les accusations d'incompétence ${ }^{30}$. Toujours est-il que les soupçons de mutilation et de destruction devaient peser lourdement sur les évolutions futures de sa carrière et entacher durablement sa postérité. Quelques années plus tard, le préfet du Rhône propose le nom de Chenavard pour succéder à Ambroise Commarmond comme inspecteur des monuments historiques: "Le département du Rhône possède des monuments historiques d'une grande importance dont l'entretien et la conservation exigent le concours simultané de l'architecte et de l'archéologue. Il m'a paru que cette double fonction serait avantageusement réunie dans les mains du nouveau conservateur. Sa nomination serait bien accueillie par l'opinion publique, unanime à louer en M. Chenavard les vertus de l'homme et la science hors ligne du savant $»^{31}$, mais le ministre de l'instruction publique et des cultes s'y oppose formellement ${ }^{32}$.

\section{La cathédrale devient l'instrument de l'État}

5 En l'espace d'une vingtaine d'années, les méthodes de restauration connaissent de profondes évolutions sous l'action des études archéologiques contemporaines et d'une lecture constructive de l'architecture médiévale. Les moyens budgétaires mis en place sous la Restauration et la Monarchie de Juillet pour l'entretien des édifices diocésains ont certes permis la réalisation d'importants travaux, mais ceux-ci ont également mis en relief les insuffisances des architectes en matière de restauration monumentale ${ }^{33}$. Cette méconnaissance ne manquera pas d'être sévèrement critiquée dès les années 1840 alors que le service des architectes diocésains se met en place, à l'image du jugement embarrassé de Labrouste à propos d'un projet (non réalisé) de stalle épiscopale pour 
Belley étudié par Chenavard: "Le reproche principal, écrit-il, le seul peut-être qu'il convienne à la commission de faire à cette composition, c'est l'oubli presque complet des principes véritables de la construction [...]. Cependant, il me parait difficile de donner des conseils de ce genre à un artiste dans la position de M. l'architecte de Belley ${ }^{34}$. On observe à ce sujet que l'architecte se retient de toute ornementation à l'exception de la façade principale qui demeure la seule partie véritablement personnalisée de la nouvelle cathédrale. Synonyme de liberté dans les premières décennies romantiques du XIX ${ }^{\mathrm{e}}$ siècle, le néo-gothique troubadour offre une fantaisie que les partisans d'un gothique "épuré par la raison» (Leniaud) s'emploieront à combattre au nom d'un esprit de système qui instrumentalisera l'archéologie nationale à des fins de cohésion du corps social et politique. Quel que soit le caractère archéologique de la reconstruction de la cathédrale de Belley, un tel chantier revêt un caractère hautement politique sous la Monarchie de Juillet comme en témoignent les discours tenus lors de la pose de la première pierre du nouvel édifice, le 14 octobre 1836, «Honneur donc, honneur à l'époque éclairée où l'État intervient dans de telles entreprises, les dirige et leur ouvre son trésor! [...] Les monuments des cultes relevés de leurs ruines, les merveilles des arts, les lumières qui moralisent, une prospérité matérielle jusqu'ici sans exemple, voilà les fruits que cette sagesse nous dispense ", s'exclame le préfet Jaÿr ${ }^{35}$. L'idée selon laquelle le néo-gothique renoue avec la période la plus prestigieuse de l'histoire nationale en accomplissant une nouvelle fois l'unité du peuple et de son gouvernement autour de la cathédrale se trouve ainsi réalisée pour la première fois en France à Belley indépendamment des travaux de Debret à Saint-Denis. La dédicace apposée lors de la cérémonie mérite également d'être soulignée: "Sur la demande réitérée de l'évêque diocésain et le concours des autorités civiles, le gouvernement a fait reconstruire ce monument, aux frais de l'État d'après les plans et devis de M. A.-M. Chenavard architecte de Lyon, en style conforme à celui du sanctuaire qui a été conservé sous le pontificat de Grégoire XVI », lit-on comme pour couper court aux critiques portant sur le caractère de la nouvelle cathédrale. Dans ce contexte, la question du couvrement de l'édifice prend une importance toute particulière (ill.2). 


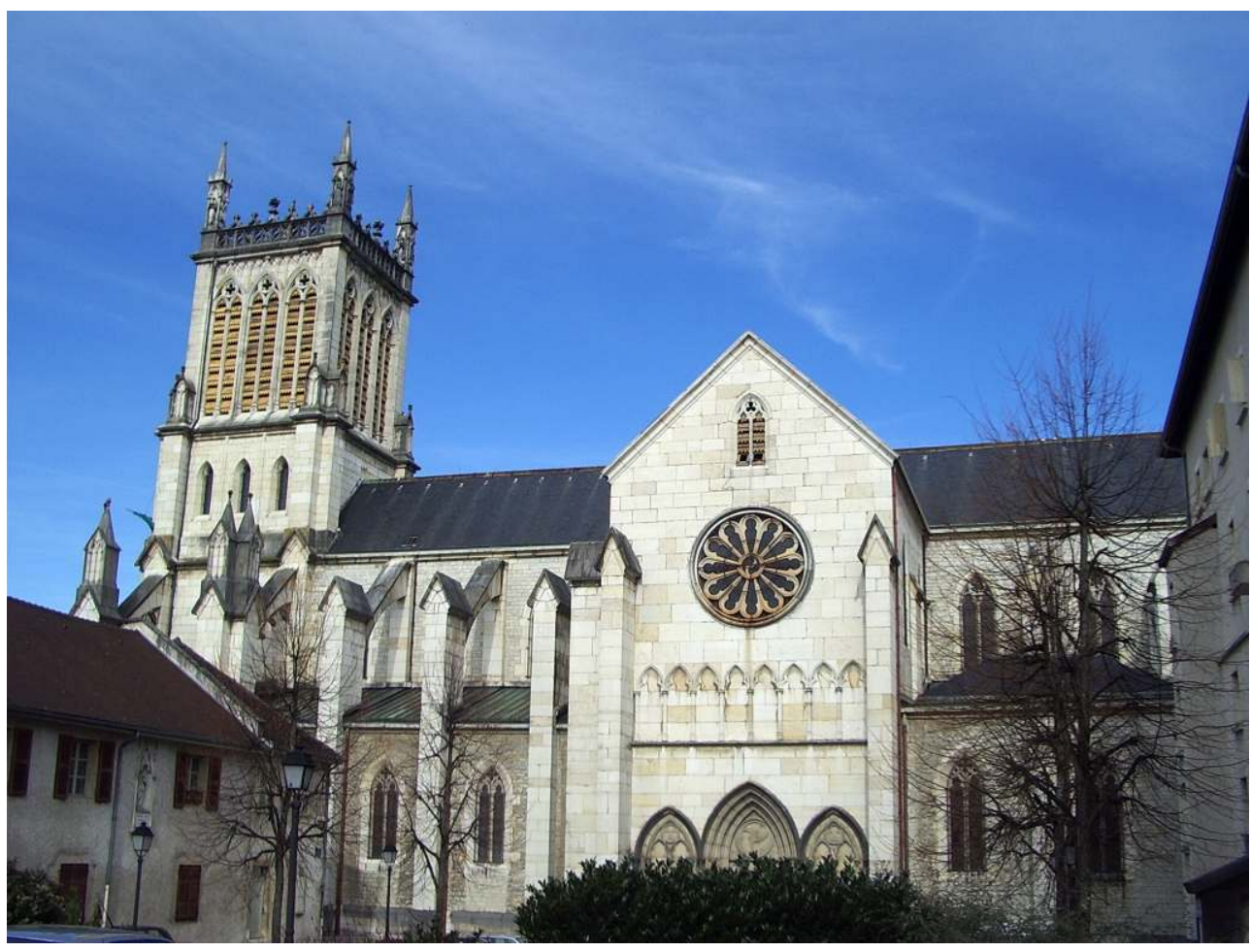

Cl. Jean-Christophe Stuccilli

6 Dans son premier projet d'achèvement, Chenavard avait songé un temps couvrir les nefs au moyen de tuiles creuses, à l'image des cathédrales de Lyon et de Vienne, mais le caractère rustique de ce mode de couvrement aurait profondément détonné avec l'abside de la cathédrale, couverte d'ardoises, le préfet de l'Ain expliquant à ce sujet que le cas de figure inverse eût été plus satisfaisant. Si la tuile creuse paraît mal adaptée au climat du département, d'autres raisons sont invoquées pour ramener la nouvelle cathédrale dans le giron de ses consœurs d'île-de-France : «À plus forte raison ne doit-on pas l'admettre pour un monument de l'État, pour une restauration qui fera époque, il ne faut pas que l'on puisse dire que ce qui appartient au siècle de Louis-Philippe est la partie la plus mal couverte [...]. Il ne faut pas que pour si peu on puisse ravaler les travaux de notre grande époque et nous reprocher ce disparate dans la restauration remarquable d'un ancien et beau monument $»^{36}$. L'idée même qu'une cathédrale gothique puisse être couverte en tuiles romaines semble incongrue et c'est précisément ce qui amènera Tony Desjardins à relever la toiture de la cathédrale Saint-Jean de Lyon en 1855 dans un essai de cathédrale idéale. Par l'ampleur des problématiques qu'elle soulève, l'affaire de la cathédrale de Belley constitue une date essentielle dans l'histoire de la restauration monumentale en France et annonce les débats passionnés qui devaient bientôt animer les acteurs de la protection du patrimoine, qu'il s'agisse de l'administration des cultes et des monuments historiques, des architectes-restaurateurs comme des figures de l'érudition locale.

Littéraire dans ses premières manifestations, l'engouement pour l'art médiéval produit dans les premières années du XIX siècle un néo-gothique pittoresque ou troubadour avant qu'à l'impulsion de Viollet-le-Duc, une archéologie raisonnée n'établisse les fondements d'une nouvelle architecture. Dans l'attente des entreprises archéologiques et littéraires des années 1840-1850, il revient à la génération des Debret et des Chenavard 
d'assurer l'entretien des monuments religieux dans une approche souvent plus décorative que véritablement structurelle et constructive. En ce domaine, Chenavard se révèle un précurseur de la théorie de l'unité de style qui marquera tant l'esprit des restaurations conduites par les figures de l'école diocésaine sous l'égide de Viollet-le-Duc et se distingue notablement de ses confrères troubadours par une approche géométrique et stéréotomique de l'architecture médiévale. Cette lecture revêt un caractère essentiellement pragmatique à l'esprit de Chenavard et s'appliquerait d'une même manière aux monuments antiques en matière d'anastylose, comme si l'architecte se retenait de toute interprétation ornementale. Il est vrai que ses tentatives en ce domaine, notamment en matière de mobilier, se sont révélées un peu hasardeuses à l'image de la chaire de la cathédrale Saint-Jean. L'achèvement de la cathédrale de Belley marque précisément le terme du premier âge néo-gothique inauguré trente ans plus tôt par la restauration de la basilique de Saint-Denis par François Debret (1820-1828), la reconstruction de la flèche de la cathédrale de Rouen par Jean-Antoine Alavoine (à partir de 1823) et l'achèvement de la cathédrale de Nantes. Le caractère du maitre d'œuvre se dévoile également à la lumière de l'affaire de Belley: intraitable, cultivant le sentiment d'avoir raison contre tous en exploitant le thème de l'incompétence de l'opinion publique, exactement à l'image de Viollet-le-Duc. C'est à la suite de son évincement à Viviers et à Lyon trois ans plus tard que l'architecte entreprendra de voyager en Orient sur les traces d'une Antiquité qui n'a jamais cessé de le fasciner et ce long séjour aux sources de son inspiration le confirmera dans ses goûts de jeunesse. De plus, les travaux néo-gothiques après 1844 sont rares même s'il est maintenu comme diocésain de Belley jusqu'en 1854 avec son élève Louis Dupasquier (1800-1870). Alors que l'architecte enchaîne les chantiers dans la région lyonnaise, à l'exception de la cathédrale Saint-Jean, la restauration des principales églises de la ville lui échappe et là réside certainement l'une des raisons pour laquelle Chenavard n'apparut jamais comme l'homme du néogothique à Lyon malgré l'importance de ses travaux; c'est à ses élèves, Desjardins et Bossan notamment, que reviendra le soin d'ouvrir la ville aux expériences archéologiques au milieu des années 1840. À Belley pourtant, leur maître inaugurera l'ère des grands vaisseaux néo-gothiques en appliquant à la construction médiévale une rigueur volumétrique toute classique dans ses assises et ses savants appareils de pierres, ces masses stéréotomiques qui le fascineront quelques années plus tard devant les remparts de l'acropole d'Athènes. Jean-Michel Leniaud n'a-t-il pas parlé du «cubisme » de la cathédrale de Belley comme pour souligner la distance qui, indéniablement, sépare l'architecte lyonnais d'un Viollet-le-Duc ou d'un Lassus ? ${ }^{37}$ Chenavard est demeuré sa vie durant un homme des Lumières aux sentiments religieux circonspects et c'est précisément en matière d'archéologie médiévale que sa bibliothèque se révèle la plus énigmatique $^{38}$. En 1860, lorsqu'il publie ses travaux et compositions, les cathédrales de Belley et de Chalon-sur-Saône figurent en bonne place aux côtés du Grand théâtre de Lyon et de son projet pour Fourvière comme si l'architecte entendait rappeler, au terme de sa carrière, la part essentielle qu'il prit dans la maturation du mouvement néomédiéval en France. 


\section{ANNEXES}

Annexe 1 - Antoine-Marie Chenavard, «Quelques observations sur le style qu'il convient de suivre dans le complément ou la restauration des édifices et dans leurs additions ", (Arch. de la société académique d'architecture de Lyon, compte rendu des séances, 7 mai 1842).

Les temps, les révolutions ruinent des monuments précieux pour les arts, beaucoup d'entre eux sont, dès leur origine, restés inachevés mais les mêmes institutions qui les avaient élevés exigent souvent qu'on les répare, qu'on exécute leur complément et quelquefois même qu'il y soit ajouté des parties que de nouveaux usages rendent nécessaires. Les incohérences que l'on remarque souvent entre les travaux auxquels on a dû se livrer et les parties anciennes de l'édifice ont donné lieu aux réflexions que nous avons l'honneur de soumettre à la société académique d'architecture désirant qu'un pareil sujet lui semble digne de fixer un instant son attention.

Quel que soit le style d'architecture d'un édifice, nous pensons qu'il y a nécessité de s'y conformer soit dans l'exécution de son complément, soit dans la restauration des parties détruites, ici l'artiste qui succède doit faire abnégation de ses propres goûts et consentir à suivre religieusement la marche tracée par le premier auteur. On ne saurait invoquer comme une raison suffisante de s'écarter de ce principe les nombreux exemples que nous ont laissés de grands maîtres dans cet art et cette multitude de détails précieux d'architecture romaine qu'ils ont exécutés dans des cathédrales gothiques, parce qu'avant l'autorité de l'exemple, il existe une loi plus impérieuse, celle de l'unité qu'il faut observer dans toute composition.

Sans doute il n'est pas rare de voir des édifices inachevés d'abord, avoir été continué dans un style différent par des hommes d'un talent supérieur et pour en citer quelques exemples : l'église de Saint-Nizier de Lyon dont l'intérieur tout entier, les faces latérales, l'abside et une partie de la face principale sont d'architecture gothique, a été continuée par Philibert Delorme dans un style romain. Lorsqu'on ignore que cette construction est l'œuvre d'un grand maître et l'un de ses essais pour arriver au développement des connaissances qu'il avait acquises en Italie, et qu'il devait mettre au jour dans les châteaux d'Anet, de Meudon, dans les tombeaux de François $1^{\mathrm{er}}$, d'Henri II enfin dans le palais des Tuileries, on éprouve un sentiment pénible en voyant le disparate des deux styles d'architecture dont le frontispice de cette église est composé.

Loin de nous cependant la pensée que l'on doit renverser aujourd'hui l'œuvre de Philibert Delorme pour y substituer une architecture plus en harmonie avec le reste de l'édifice, il y a quelque chose de plus précieux que la restauration gothique d'un moderne sans consistance, c'est la trace du passage d'un grand maître. Dans cet art est l'un des documents qu'il nous a laissés pour apprendre à marcher dans la carrière où il s'est illustré.

Saint Gervais à Paris offre aussi un exemple de ce genre de disparate malgré le talent qui brille dans la superposition des trois ordres d'architecture romaine de son portail. Qui 
n'éprouve pas en entrant sous ces nefs gothiques une sensation désagréable produite par cette discordante transition?

Mais si la même architecture doit être observée dans tout ce qui forme le complément ou la restauration d'un édifice, il n'en est pas ainsi des ouvrages accessoires qu'un architecte peut être appelé à exécuter dans un monument d'une architecture qui n'est plus celle de nos jours. Ici, nous semble se présenter une distinction substantielle, unité à observer dans toutes les parties constructives et dans celles qui ne sont que leur complément sans lesquelles l'édifice ne s'offre aux regards que comme un corps mutilé, variété permise dans les détails qui ne sont pas inhérents à sa construction primitive et qui n'en font point partie intégrante tels que les décors d'une chapelle et toute addition étrangère à la composition première d'un monument. Dans ce cas, s'il se présente quelques difficultés à continuer le style du monument ou si un penchant particulier porte l'architecte à s'éloigner du genre adopté dans le reste de l'édifice, une entière liberté doit lui être laissée ; s'il prend un style différent, il ne pèche point contre l'unité, car ce qu'il introduit est une œuvre détachée non prévue par le premier auteur, amenée par de nouvelles idées, de nouveaux besoins et qui n'importent point à son complément. Nous devons applaudir au contraire à la composition faite dans un style pratiqué à l'époque où ce second artiste a vécu car il a respecté la première œuvre, il n'a point voulu la dénaturer et par une imitation plus ou moins parfaite tromper les siècles à venir et enfin parce qu'il a du moins posé un caractère [illisible], un jalon dans l'histoire de l'art.

Notre opinion serait toutefois bien différente s'il s'agissait d'élever un tombeau pour recevoir les cendres depuis longtemps refroidies d'un homme illustre. Nos respects s'attachent à la pierre comme aux cendres elles-mêmes, en les réunissant pour toujours, qui ne veut plus les séparer, s'accoutume à confondre ensemble ces deux objets de notre vénération et veut retrouver dans cette silencieuse demeure l'aspect du temps ou l'homme à vécu.

Pour avoir été méconnu ce sentiment, qui pourra reconnaitre le tombeau de Dagobert mort au VII siècle, époque de l'architecture romaine du bas empire, dans le tombeau gothique qui lui fut élevé six siècles plus tard, là le bon goût repousse tout.

L'esprit cherche en vain l'image qui s'est formée sous les traits qui n'ont pu lui appartenir, là il y a nécessité absolue de s'identifier avec le caractère de l'art au temps ou vivait l'homme dont le monument rappelle la mémoire, alors l'artiste habile doit être aussi antiquaire profond.

Ainsi donc, si les lois de l'unité exigent que toutes les restaurations des parties constitutives d'un édifice ou l'exécution d'un complément [soient] du même style que l'objet restauré, nous pensons que [si] dans des dispositions accessoires, étrangères à leur composition première, on fait suivre la marche des arts et prendre un style différent de celui du reste du monument, la raison ne s'y oppose point. Il y a avantage pour l'histoire de l'art et, en diverses rencontres, le goût peut le recommander.

\section{NOTES}

1. 1. Arch. départ. du Rhône, 3 V 19, lettre de Chenavard au préfet du Rhône [à propos de la cathédrale de Lyon], 8 mai 1838. 
2. 2. Antonin Louvier et Adolphe Coquet, « Notice historique sur la vie et les œuvres d'AntoineMarie Chenavard architecte [...] », Annales de la Société académique d'architecture de Lyon, t. VIII, [1883-1886], 1887, p. 1-17.

3. 3. Clair Tisseur, Antoine Chenavard, discours de réception prononcé dans la séance publique du 21 juin 1887 à l'Académie des sciences, belles-lettres et arts de Lyon, Lyon, Henri Georg, 1887, 38 p.

4. 4. Jean Roidot, M. Chenavard et ses œuvres. Notice lue à la Société éduenne, 1862, p. 426.

5. 5. [Anonyme], « Notice nécrologique d'Antoine-Marie Chenavard », L'Écho de Fourvière, 5 janvier 1884, p. 11-12.

6. 6. Annales archéologiques, t. I, 1844, p. 135.

7. 7. Antoine-Marie Chenavard, Sur le goût dans les arts, discours prononcé à l'Académie royale de Lyon le 14 juillet 1831, Lyon, 1831, p. 15.

8. 8. Ibid., p. 6.

9. 9. Mgr Alexandre-Raymond Devie, Rituel de Belley, Manuel des connaissances utiles aux ecclésiastiques sur divers objets d'art [...], Bourg, 1830-1831, 4 vol.

10. 10. Chiffres cités par Charles Dementhon, La Cathédrale de Belley, histoire et description, Paris, Beauchesne, Lyon, Lardanchet, 1916, p. 187.

11. 11. Arch. départ. de l'Ain, 3 V12, lettre de Mgr Devie au ministre des cultes, 7 avril 1829.

12. 12. Arch. départ. de l'Ain, 3 V12, rapport du conseil des bâtiments civils, 6 juin 1829.

13. 13. Arch. départ. de l'Ain, $3 \mathrm{~V} 12$, lettre du ministre des affaires ecclésiastiques et de l'instruction publique au préfet de l'Ain, 22 avril 1830.

14. 14. Arch. départ. de l'Ain, 3 V12, lettre de Mgr Devie au préfet de l'Ain, 18 octobre 1832.

15. 15. Arch. départ. de l'Ain, 3 V14, rapport adressé par Chenavard au ministre des cultes, 7 novembre 1833.

16. 16. Arch. nat., $\mathrm{F}^{19} 7640$, rapport de Chenavard au préfet de l'Ain, 6 juin 1836.

17. 17. Arch. nat., $\mathrm{F}^{19} 7640$, rapport de Caristie au conseil des bâtiments civils, 5 décembre 1839.

18. 18. Arch. nat., $\mathrm{F}^{19} 7640$, lettre du directeur de l'administration des cultes au ministre des cultes, 11 novembre 1839 .

19. 19. Arch. nat., $\mathrm{F}^{19} 7641 / 2$, propos de Ferdinand de Guilhermy, cités par Chenavard, "Copie d'un article adressé au rédacteur du Courrier de l'Ain en réponse à divers passages d'un rapport fait par Mr de Guillermi [sic] à monsieur le ministre de l'instruction publique sur la cathédrale de Belley et celle de Lyon », 28 septembre 1838.

20. 20. Ibid.

21. 21. Ibid.

22. 22. Antoine-Marie Chenavard, "Quelques observations sur le style qu'il convient de suivre dans le complément ou la restauration des édifices et dans leurs additions ", (7 mai 1842), cf. annexe 1.

23. 23. Arch. nat., $\mathrm{F}^{19} 7640$, rapport de l'architecte de la cathédrale de Belley au ministre des cultes, 30 avril 1850.

24. 24. Arch. nat., $\mathrm{F}^{19} 7640$, rapport de la commission des arts et édifices religieux, 2 septembre 1848.

25. 25. Arch. nat., $\mathrm{F}^{19} 7641 / 2$, rapport de Viollet-le-Duc à la commission des arts et des édifices religieux, 27 septembre 1848.

26. 26. Arch. nat., $\mathrm{F}^{19} 7640$, rapport de la commission des arts et des édifices religieux, 20 juin 1850.

27. 27. Arch. nat., $\mathrm{F}^{19} 7640$, rapport de l'architecte de la cathédrale de Belley au ministre des cultes, 30 avril 1850.

28. 28. Arch. départ. de l'Ain, 3 V14, lettre de Chenavard au ministre des cultes, 9 septembre 1850. 
29. 29. Arch. départ. de l'Ain, 3 V14, lettre du ministre des cultes au préfet de l'Ain, 14 septembre 1850.

30. 30. Arch. nat. $\mathrm{F}^{19} 7641 / 2$, réponse de l'architecte de la cathédrale de Belley aux questions contenues dans la lettre au ministre de l'instruction publique et des cultes du 20 juillet 1848, 2 septembre 1848.

31. 31. Arch. départ. du Rhône, $4 \mathrm{~T}$ 8, lettre du préfet du Rhône au ministre de l'instruction publique et des cultes, 26 septembre 1857.

32. 32. Arch. départ. du Rhône, $4 \mathrm{~T}$ 8, lettre du ministre de l'instruction publique et des cultes au préfet du Rhône, $1^{\mathrm{er}}$ juin 1858.

33. 33. Jean-Michel Leniaud, Les Cathédrales au XIXe siècle. Étude du service des édifices diocésains, Paris, Economica, 1993, p. 254 et suivantes.

34. 34. Arch. nat., $\mathrm{F}^{19} 7640$, rapport de Labrouste à la commission des arts et édifices religieux, 16 décembre 1852.

35. 35. Cité par M. Depéry, Cathédrale de Belley, de sa reconstruction, Belley, Verpillon, 1836, p. 36.

36. 36. Arch. nat., $\mathrm{F}^{19} 7640$, lettre du sous-préfet de Belley au préfet de l'Ain, 15 avril 1844.

37. 37. Jean-Michel Leniaud, "Du néo-classique au béton », dans Ces Églises du dix-neuvième siècle (en collaboration), Amiens, Encrage, 1993, p. 46.

38. 38. Philippe Dufieux, «La bibliothèque d'Antoine-Marie Chenavard (1787-1883) », Livraisons d'histoire de l'architecture, $\mathrm{n}^{\circ} 15,2008, \mathrm{p} .101-118$.

\section{RÉSUMÉS}

L'allégeance d'Antoine-Marie Chenavard (1787-1883) à la culture classique a longtemps occulté les travaux de l'architecte en matière de restauration monumentale, lui qui compte pour l'une des figures majeures du gothique troubadour en France dont la cathédrale de Belley (1836-1855), premier essai de cathédrale idéale avant les entreprises archéologiques de Lassus et de Viollet-leDuc, constitue l'une des réalisations les plus emblématiques. Par l'ampleur des problématiques qu'il soulève, ce dernier chantier constitue une date essentielle pour l'histoire de la restauration monumentale en France et marque le terme du premier âge néo-gothique inauguré trente ans plus tôt en France par la restauration de la basilique de Saint-Denis par François Debret (1820-1828), la reconstruction de la flèche de la cathédrale de Rouen par Jean-Antoine Alavoine (à partir de 1823) et l'achèvement de la cathédrale de Nantes.

The submission of Antoine-Marie Chenavard (1787-1833) to classical culture has hidden for a long time the restoration works of the architect on monuments, for someone who counts as one of the major figures of the "trobadour " gothic architecture in France, including the Belley cathedral, first attempt of an ideal cathedral before the archeological works of Lassus and Viollet-le-Duc, and which is one of the most emblematic buildings. Because of the huge amount of problematics contained within this work, this last work is a crucial moment in the history of the restoration of monuments in France, and marks the end of the first period of neo-gothic architecture, inaugurated thirty years before in France with the restoration of the Saint-Denis basilica by François Debret, the rebuilding of the spire of the Rouen cathedral by Jean-Antoine Alavoine (from 1823) and the completion of the Nantes cathedral. 
Die besondere Vorliebe Antoine-Marie Chenavards (1787-1883) für die Klassik hat die Arbeiten des Architekten im Bereich der Restaurierung von Monumenten lange im Hintergrund gelassen. Chenavard zählt aber zu den Hauptanhängern des Troubadour-Stils in Frankreich. Schon vor den archäologischen Werken Lassus' und Viollet-le-Ducs führt eines seiner repräsentativsten Werke, nämlich die Kathedralkirche von Belley (1836-1855), den ersten Versuch einer idealen Kathedrale vor Augen. So erweist sich dieses Bauwerk ganz wesentlich in der Geschichte der monumentalen Restaurierung, indem es eine weitgehende Problematik verdeutlicht. Es bedeutet also das Ende der ersten Zeit der Neogotik, die dreißig Jahre früher in Frankreich eingeleitet wurde, und zwar mit der zwischen 1820 und 1828 von François Debret durchgeführten Restaurierung der Basilika Saint-Denis, dann mit dem Wiederaufbau der Turmspitze des Domes in Rouen von Jean-Antoine Alavoine ab 1823 und schließlich mit der Fertigstellung der Kathedrale von Nantes.

\section{AUTEUR}

\section{PHILIPPE DUFIEUX}

Philippe Dufieux est docteur de l'École pratique des hautes études (section des sciences historiques et philologiques), diplômé en histoire, histoire de l'art et en sciences politiques. Il a été directeur de projets au sein de la direction de l'aménagement urbain de la ville de Lyon. Depuis 2003, il est chargé de mission auprès du conseil d'architecture, d'urbanisme et de l'environnement du Rhône. Vice-président de la société historique, archéologique et littéraire de Lyon, il a été chargé de cours à l'université Lyon 2. Il s'est illustré par de nombreux travaux sur l'art à Lyon aux XIX ${ }^{\mathrm{e}}$ et $\mathrm{XX}^{\mathrm{e}}$ siècles, dont Le Mythe de la primatie des Gaules. Pierre Bossan (1815-1888) et l'architecture religieuse en Lyonnais au XIX ${ }^{e}$ siècle aux Presses universitaires de Lyon (2004, préface de Jean-Michel Leniaud), Le Rêve de la maison. Cités-jardins, lotissements et habitat durable dans le Rhône, paru aux Éditions du CAUE du Rhône (2007) et Sculpteurs et architectes à Lyon (1910-1960) de Tony Garnier à Louis Bertola aux Éditions Mémoire active (2007, préface de Jean-Michel Leniaud). En 2008, il a assuré la coordination d'un numéro de la revue Livraisons d'histoire de l'architecture consacré aux chambres de commerce. Il a participé à de nombreux colloques internationaux dont Lyon-Genève-Paris (2002), Henri Focillon (2004), Les Orientalismes en architecture (2006), Villes et religions dans l'Europe moderne et contemporaine (2006), La Colline de Chaillot et ses palais (2006) et Le Livre et l'architecte (2008). Il prépare actuellement une étude sur les élèves de Tony Garnier et l'École régionale d'architecture de Lyon ainsi qu'une monographie sur l'architecte Antoine-Marie Chenavard (1797-1883). Il enseigne actuellement à l'École nationale supérieure d'architecture de Lyon. Adresse électronique : ph.dufieux@caue69.fr 